\title{
RELAÇÃO ENTRE O ESTADIAMENTO,O TRATAMENTO E A SOBREVIDA NO CÂNCER DA FARINGE
}

\author{
RELATIONSHIP BETWEEN STAGE, TREATMENT AND SURVIVAL FOR PHARYNX \\ CANCER
}

\author{
José Carlos Berto'; Abrão Rapoport, ECBC-SP²; Carlos Neutzling Lehn²; \\ Guilherme Antonio Cestari Filho' ${ }^{1}$ Afonso do Carmo Javaroni ${ }^{1}$
}

\begin{abstract}
RESUMO: Objetivo: Estudo da sobrevida de pacientes portadores de carcinoma epidermóide (CEC) da faringe a partir do estadiamento e tratamento isolado (cirurgia ou radioterapia) e associação terapêutica (cirurgia e radioterapia ou quimioterapia). Métodos: consideradas variáveis como gênero, etnia, sintomas, estadiamento, tratamento e sobrevida, foram utilizados para cálculo da sobrevida o método de Kaplan Meyer e para verificar a associação de variáveis o teste do Qui Quadrado ( $<<0,05)$. Resultados: A idade média foi de 56,5 anos ( \pm 11,9 anos), sendo 456 homens e 51 mulheres (relação 9:1). Quanto à etnia, a branca foi constatada em 480 pacientes $(94.7 \%)$ e a negra em 27(5.3\%) com relação de 19:1. Quanto à sintomatologia, ferida na garganta (13.6\%), linfonodo metastático cervical (11.8\%) e rouquidão (7.9\%). Quanto ao estadiamento, o estádio IV predominou (60.6\%), o que justificou a indicação de cirurgia isolada, em $11.0 \%$ dos casos e da radioterapia isolada em $5.7 \%$. No que diz respeito à sobrevida, tivemos com a cirurgia isolada para a hipofaringe $62.2 \%$ e $68.0 \%$ para a orofaringe, e $38.9 \%$ para a nasofaringe com a quimioterapia associada à radioterapia. Conclusão: A sobrevida a cinco anos foi satisfatória nos estádios iniciais na oro e hipofaringe com o tratamento cirúrgico e na nasofaringe com o tratamento quimioterápico associada à radioterapia (Rev. Col. Bras. Cir. 2006; 33(4): 207-210).
\end{abstract}

Descritores: Neoplasias faríngeas; Sobrevida; Estadiamento de neoplasias; Terapia combinada; Carcinoma de células escamosas

\section{INTRODUÇÃO}

As neoplasias malignas da faringe são freqüentes dentre aquelas que acometem as vias aerodigestivas superiores, tendo como fatores de risco o álcool e o fumo ${ }^{1}$ são predominantes em homens na quinta década de vida ${ }^{2,3}$, com grande predomínio do carcinoma espinocelular (CEC) em 95\% dos $\operatorname{casos}^{4,5}$. Na etiopatogenia do câncer da faringe, o tabaco é fator de risco em $75 \%$ dos $\operatorname{casos}^{6}$ decorrente do alcatrão como um dos seus componentes onde o benzopireno é um potente agente cancerígeno ${ }^{7}$. Já o álcool, co-carcinógeno responsável pelo reparo do $\mathrm{DNA}^{5,8,9}$, contém altas concentrações de etanol que diminui a secreção salivar com conseqüente aumento da concentração de carcinógenos ${ }^{1,9,10}$.

Outro aspecto que merece especial atenção, é o diagnóstico tardio do CEC da faringe, quando as neoplasias somente são diagnosticadas a partir do estádio clínico II, com tempo médio de queixa de 17 meses, com tempo de sobrevida médio de $41.8 \%$ para o estádio III e $22.0 \%$ para o estádio IV, sendo os melhores resultados com a associação da operação e radioterapia em relação à radioterapia isolada ${ }^{11}$.

Considerados os aspectos acima, procuramos correlacionar o estadiamento do câncer da faringe com o estadiamento e a sobrevida nas diferentes fases desta doença neoplásica.

\section{MÉTODO}

Foram revistos prontuários de 507 pacientes portadores de CEC de faringe (hipofaringe, orofaringe e nasofaringe) no Departamento de Cirurgia de Cabeça e Pescoço ao Hospital Amaral Carvalho, Jaú, de 1996 a 2001.

Esta casuística foi analisada segundo variáveis como gênero, etnia, estádio clínico, tratamento realizado e sobrevida.

Para análise da sobrevida a cinco anos e o tempo mediano da mesma, foi utilizado o método Kaplan-Meier (1958), enquanto que para verificar a associação de variáveis, empregamos o teste do qui-quadrado $(\mathrm{p}<0,05)$.

\section{RESULTADOS}

Foram analisados 507 prontuários de pacientes com CEC de faringe com idade média de 56,5 anos \pm 11.9 anos, variando de 11 a 87 anos, 456 homens e 51 mulheres (relação 9:1). Quanto à etnia, a branca foi presente em 480 casos (94.7\%) e a negra em 27 casos (5,3\%) com relação de 19:1.

Quanto à sintomatologia, a odinofagia predominou como primeiro sintoma (66.7\%) seguido de ferida na garganta (13.6\%), linfonodo cervical $(11.8 \%)$ e rouquidão $(7.9 \%)$ respectivamente (Tabela 1).

1. Médico cirurgião do Hospital Amaral Carvalho, Jaú, São Paulo.

2. Médico cirurgião do Hospital Heliópolis, Hosphel, São Paulo.

Recebido em 15-02-06

Aceito para publicação em 16-03-06

Conflito de interesses: nenhum

Fonte de financiamento: nenhuma

Trabalho realizado no Hospital Amaral Carvalho, Jaú, São Paulo. 
Tabela 1 - Distribuição das principais queixas do paciente quando da primeira consulta médica.

\begin{tabular}{|c|c|c|c|c|}
\hline & Hipofaringe $\mathrm{N}(\%)$ & Orofaringe N (\%) & Nasofaringe $\mathrm{N}(\%)$ & Total N (\%) \\
\hline Odinofagia & $109(68,6)$ & $228 \quad(72,8)$ & $1 \quad(2,9)$ & $338(66,7)$ \\
\hline Ferida na boca & 0 & $68 \quad(21,8)$ & $1 \quad(2,9)$ & $69(13,6)$ \\
\hline Linfonodo cervical & $12 \quad(7,5)$ & $15 \quad(4,8)$ & $33(94,2)$ & $60(11,8)$ \\
\hline Rouquidão & $38 \quad(23,9)$ & $2 \quad(0,6)$ & 0 & $40 \quad(7,9)$ \\
\hline Total & $159(31,4 \%)$ & $313(61,8 \%)$ & $35 \quad(6,8 \%)$ & $\mathbf{5 0 7}$ \\
\hline
\end{tabular}

$C^{2}=354,924 ; p<0,0001$.

Quanto ao estadiamento (Tabela 2), o estádio III foi diagnosticado em $29,5 \%$ na hipofaringe e em $26,8 \%$ na orofaringe, enquanto que o estádio IV $67,3 \%$ na hipofaringe e $62.3 \%$ na orofaringe, enquanto que no câncer da nasofaringe o estádio IV predominou em $57.2 \%$. Na análise de toda a casuística, as neoplasias da faringe incidiram em 322 pacientes no estádio IV $(63.5 \%)$ e em 135 no estádio III $(26,6 \%)$, sendo que o diagnóstico mais precoce somente foi possível em 50 $\operatorname{casos}(9.9 \%)$.

No que diz respeito aos tratamentos realizados nos pacientes de acordo com o sítio anatômico da doença, para o câncer da hipofaringe, 33,3\% dos pacientes foram tratados com radioterapia ( 6.000 cGy na lesão primária e 5.000 cGy no pescoço) e quimioterapia, seguidos pelo tratamento cirúrgico e radioterápico em $15,1 \%$ destes casos. Para o câncer da orofaringe, $37,4 \%$ dos casos foram tratados com radioterapia (6.000 cGy na lesão primária e 5.000 cGy no pescoço) e quimioterapia (cisplatinum e fluoracil a cada três semanas) onde $19,2 \%$ destes pacientes receberam quimioterapia isolada como paliação. No câncer da nasofaringe, $54,2 \%$ dos pacien- tes receberam radioterapia e quimioterapia (cisplatinum e fluoracil a cada três semanas ) e $25,7 \%$ somente quimioterapia paliativa. Da amostra estudada, observou-se que $11 \%$ do total de pacientes foram submetidos somente ao tratamento operatório e 3,6\% não receberam nenhum tratamento. Radioterapia exclusiva foi realizada em 5,7\% do total de pacientes tratados e foram empregadas as três modalidades de tratamento em $11,4 \%$ do total de pacientes (Tabela 3 ), percebendo-se a grande variedade de associações terapêuticas decorrentes dos estádios avançados das neoplasias da faringe.

Quanto à sobrevida dos pacientes, a estratificação em estádios não foi possível por considerarmos a pobreza na sobrevida, o que não permite a análise evolutiva a partir do TNM. Para os pacientes com neoplasia da hipofaringe de acordo com o tipo de tratamento realizado isoladamente, observou-se que nos pacientes tratados exclusivamente com radioterapia a sobrevida máxima foi de 47,4 meses e o tempo mediano de sobrevivência situou-se em 13,9 meses. Nos pacientes tratados com quimioterapia o tempo máximo de sobrevida foi de 27,1 meses e o tempo mediano de sobrevi-

Tabela 2 - Distribuição do estádio da doença nas diferentes localizações dos tumores, no momento do diagnóstico.

\begin{tabular}{|c|c|c|c|c|}
\hline Estadiamento & Hipofaringe $\mathrm{N}(\%)$ & Orofaringe $\mathbf{N}(\%)$ & Nasofaringe $N(\%)$ & Total N (\%) \\
\hline I & - & $5 \quad(1,6)$ & - & $\begin{array}{ll}5 & (1,0)\end{array}$ \\
\hline II & $5 \quad(3,2)$ & $29 \quad(9,3)$ & $11(31,4)$ & $45 \quad(8,9)$ \\
\hline III & $47 \quad(29,5)$ & $84(26,8)$ & $4(11,4)$ & $135(26,6)$ \\
\hline IV & $107 \quad(67,3)$ & $195(62,3)$ & $20(57,2)$ & $322(63,5)$ \\
\hline TOTAL & $159(31,4 \%)$ & $313(61,8 \%)$ & $35(6,8 \%)$ & 507 \\
\hline
\end{tabular}

$C^{2}=33,303, p<0,05$.

Tabela 3 - Distribuição dos tratamentos realizados nos pacientes.

\begin{tabular}{lrrrrrrrr}
\hline & Hipofaringe N(\%) & Orofaringe N(\%) & Nasofaringe N(\%) & \multicolumn{2}{c}{ Total N(\%) } \\
\hline Sem Tratamento & 5 & $(3,1)$ & 13 & $(4,2)$ & 0 & & 18 & $(3,6)$ \\
Tratamento operatório & 21 & $(13,2)$ & 35 & $(11,2)$ & 0 & & 56 & $(11)$ \\
Radioterapia & 5 & $(3,1)$ & 23 & $(7,3)$ & 1 & $(2,9)$ & $\mathbf{2 9}$ & $(\mathbf{5 , 7})$ \\
Quimioterapia & 23 & $(14,5)$ & 60 & $(19,2)$ & 9 & $(25,7)$ & $\mathbf{9 2}$ & $(\mathbf{1 8 , 1 )}$ \\
Radioterapia/tratamento operatório & 24 & $(15,1)$ & 24 & $(7,6)$ & 1 & $(2,9)$ & $\mathbf{4 9}$ & $\mathbf{( 9 , 7 )}$ \\
Quimioterapia/tratamento operatório & 5 & $(3,2)$ & 11 & $(3,5)$ & 0 & & 16 & $(3,2)$ \\
Radioterapia/quimioterapia & 53 & $(33,3)$ & 117 & $(37,4)$ & 19 & $(54,2)$ & $\mathbf{1 8 9}$ & $\mathbf{( 3 7 , 3 )}$ \\
Radioterapia/quimioterapia e tratamento operatório & 23 & $(14,5)$ & 30 & $(9,6)$ & 5 & $(14,3)$ & $\mathbf{5 8}$ & $\mathbf{( 1 1 , 4 )}$ \\
Total & $\mathbf{1 5 9}$ & $\mathbf{3 1 3}$ & & $\mathbf{3 5}$ & $\mathbf{5 0 7}$ & \\
\hline
\end{tabular}


vência foi de 6,3 meses, enquanto nos pacientes cujo tratamento foi apenas cirúrgico, a sobrevida em 5 anos foi de $62,2 \%$, havendo diferença estatística entre as curvas de sobrevida (Figura 1).

Quanto à sobrevida dos pacientes com neoplasia da orofaringe, de acordo com o tipo de tratamento realizado isoladamente, observou-se que nos pacientes tratados exclusivamente com radioterapia, o tempo máximo de seguimento foi de 42,5 meses. A taxa de sobrevida neste período foi de $17,4 \%$ e o tempo mediano de sobrevivência 8,2 meses. Nos pacientes tratados com quimioterapia o tempo máximo de sobrevida foi de 31,0 meses e o tempo mediano de sobrevivência 4,8 meses; enquanto nos pacientes no qual o tratamento foi apenas cirúrgico a taxa de sobrevida foi de $68,0 \%$ para 53,5 meses, tempo máximo de seguimento. Houve diferença estatística entre as curvas (Figura 2).

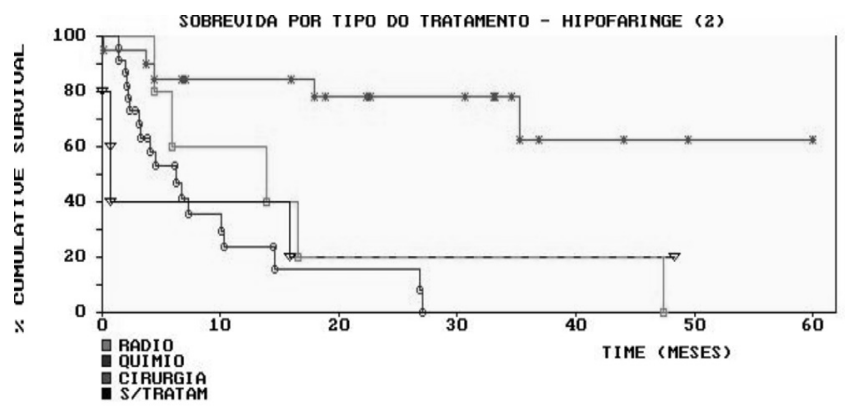

Figura 1 - Sobrevida dos pacientes com neoplasia da hipofaringe de acordo com o tipo tratamento realizado isoladamente.

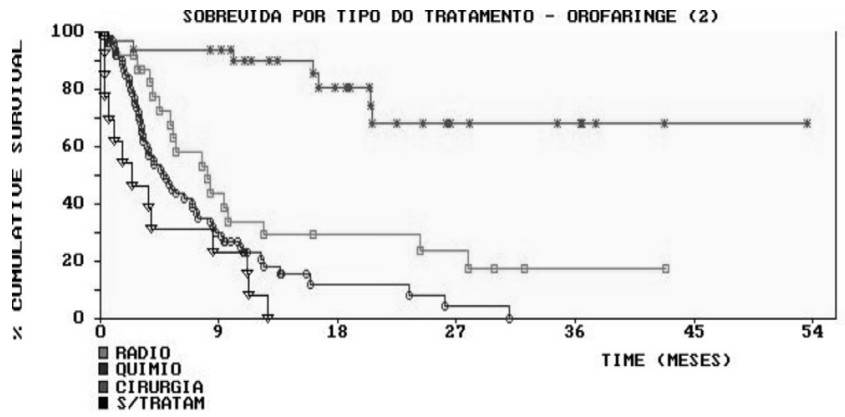

Figura 2 - Sobrevida dos pacientes com neoplasia da orofaringe de acordo com o tipo tratamento realizado isoladamente.

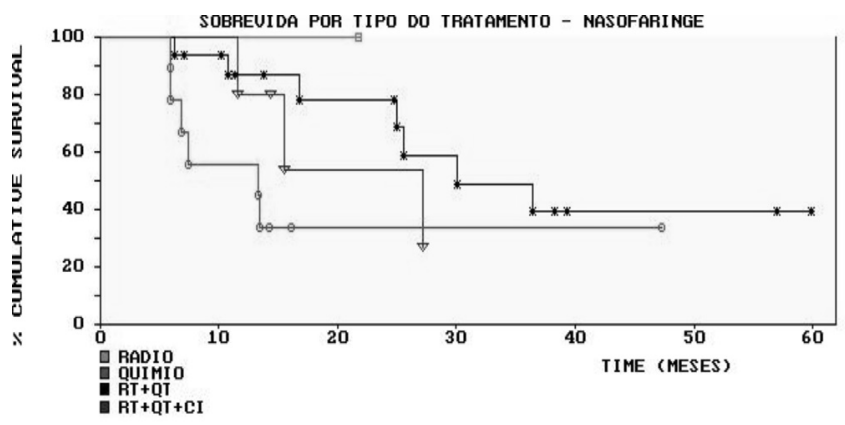

Figura 3 - Sobrevida dos pacientes com neoplasia da nasofaringe de acordo com o tipo do tratamento realizado.
Finalmente, a análise da sobrevida no câncer da nasofaringe (Figura 3), a sobrevida na associação da quimioterapia a cinco anos foi de $38,9 \%$, com tempo mediano de 30,1 meses e na associação destas à cirurgia 26,7\% dos pacientes sobreviveram 27.2 meses.

\section{DISCUSSÃO}

As neoplasias da faringe cursam com sintomatologia inicial de forma insidiosa e tardia (Tabela 1), justificando via de regra, o retardo diagnóstico (clínico e histológico) na fase avançada (estádios III e IV). Assim, a odinofagia foi o sintoma predominante $(66.7 \%)$, seguida da metástase linfonodal $(11.8 \%)$, coincidente com a literatu$\mathrm{ra}^{12}$. Quanto à distribuição anatômica, a maior freqüência da orofaringe $(61.8 \%)$ em relação à hipofaringe $(31.4 \%)$ e à nasofaringe $(6.8 \%)$ foi idêntica a de outras séries históri$\operatorname{cas}^{13-15}$

No que diz respeito ao estadiamento (Tabela 2), os estádios III e IV (avançados) foram os habituais para a hipofaringe, orofaringe $(96.8 \%, 89.10 \%$ e $68.6 \%)$ respectivamente, coincidente com a literatura 5,16 . Tais aspectos merecem uma reflexão sobre a importância do diagnóstico precoce para o câncer da faringe. Enquanto para a hipo e orofaringe nem sempre é possível, para a nasofaringe, a sintomatologia mais evidente aliada a neoplasias epidermóides menos diferenciadas e sensíveis à irradiação, permitiu planejamento terapêutico de maior sucesso.

Para o planejamento terapêutico (Tabela 3), houve nítida indicação da associação terapêutica (quimioterápica), como em todos os relatos ${ }^{2,4,5}$, para os estádios avançados(III e IV). Percebe-se pela sintomatologia que o predomínio dos estádios avançados na oro e hipofaringe justificam a indicação das irradiações em maior número de forma isolada ou associada à quimioterapia. A cirurgia isolada ou associada à radioterapia no pós operatório é reservada para os pacientes nos estádios I, II e alguns III. A quimioterapia isolada deve ser empregada nos casos de paliação.

Quanto à análise da sobrevida no câncer da faringe (Figuras 1, 2 e 3), para a hipofaringe o tratamento de eleição foi o cirúrgico isolado $(62.2 \%$ de sobrevida a cinco anos) na orofaringe $(68.0 \%$ de sobrevida a cinco anos) e para a nasofaringe a associação da quimioradioterapia (38.9\% de sobrevida a cinco anos), resultados estes concordantes com a literatura ${ }^{5,15,16}$. Todavia, conclui-se que a sobrevida é função da precocidade do diagnóstico e da presteza na indicação terapêutica para o câncer da faringe.

Em síntese, a sobrevida no câncer da faringe é satisfatória nos estádio iniciais, quando são empregados terapias isoladas, cirurgia ou radioterapia para a oro e hipofaringe sendo reservada a associação quimioradioterápica como melhor forma de tratamento para a nasofaringe. Outras formas de associações são dependentes do estádio clínico de cada paciente, que habitualmente apresentam comorbidades clínicas (pneumopatias e hepatopatias). 


\begin{abstract}
Background: Our purpose is to establish the relationship between stage, therapy and survival for patients with squamous cell carcinoma (SCC) of the pharynx. Methods: A retrospective study of 507 files of patients with squamous cell carcinoma (SCC) of the phrarynx at Department de Cirurgia de Cabeça e Pescoço do Hospital Amaral Carvalho, Jaú, from 1996 to 2001 was conduced. Considering Variables such as gender, ethnic, symptons, staging, therapy and survival were evaluated using Kaplan Meyer Method for survival and K Square Test $(p<0,05)$ for others variables. Results: Median age was 55,6 years $( \pm 11,9$ years), 456 males and 51 females (9:1 relation). About ethnic, 480 patients were white (94.7\%) and 27 black, follow by wound (13.6\%), neck node (11.8\%) and hoaseness (7.9\%). Stage IV (60.6\%), justifyng isolated surgery in $11.0 \%$ and irradiation in $5.7 \%$. For hipopharynx cancer with surgery alonemortality was (62.2\%), for oropharynx (68.0\%) and nasopharynx (38.9\%) with chemoradiotherapy association. Conclusion:The 5 years survival for initial stages for oropharynx and hipopharynx were satisfactory with surgical therapy and for nasopharynx with chemoradiotherapy association.
\end{abstract}

Key words: Pharyngeal neoplasms; Survivorship; Neoplasm staging; Combined modality therapy; Carcinoma, squamous cell.

\section{REFERÊNCIAS}

1. Maier H, Dietz A, Gewelke U, Heller WD, Weidaver H. Tobacco and alcohol and the risk of head and neck cancer. Clin Invest. 1992;70(3-4):320-7.

2. Johansen LV, Grau C, Overgaard J. Hypopharyngeal squamous cell carcinoma: treatment results in 138 consecutively admitted patients. Acta Oncol. 2000;39(4):529-36.

3. Prout MN, Sidari JN, Witzburg RA, Grillone GA, Vaughan CW. Head and neck cancer screening among 4611 tobacco users older than forty years. Otolaryngol Head Neck Surg. 1997;116(2):2018 .

4. Dedivitis RA. Etiologia do carcinoma epidermóide de hipofaringe. Rev Bras Cancerol. 2000;46(2):183-9.

5. Muir C, Weiland L. Upper aerodigestive tract cancers. Cancer. 1995;75(1 Suppl.):147-53. Erratum in: Cancer 1995;75(12):2978.

6. Johnson N. Tobacco use and oral cancer: a global perspective. J Dental Educ. 2001;65(4):328-39.

7. International Agency for Research on Cancer (IARC). Tobacco smoking. Monogr. Eval Carcinog Risk Hum 38; Lyon: IARC; 1986.

8. Schottenfeld D. Alcohol as a co-factor in the etiology of cancer. Cancer. 1979; 43(5 Suppl.):1962-6.

9. Leclerc A, Brugère J, Luce D, Point D, Guenel P. Type of alcoholic beverage and cancer of the upper respiratory and digestive tract. Eur J Cancer Clin Oncol. 1987;23(5):529-34.

10. Rothman K, Keller A. The effect of joint exposure to alcohol and tobacco on the risk of cancer of the mouth and pharynx. J Chronic Dis. 1972;25(12):711-6.
11. Guggenheimer J, Verbin RS, Johnson JT, Horkowitz CA, Myers EN. Factors delaying the diagnosis of oral and oropharyngeal carcinomas. Cancer. 1989;64(4): 932-5.

12. Spitz MR, Fueger JJ, Goepfert H, Hong WK, Newell GR. Squamous cell carcinoma of the upper aerodigestive tract. A case comparison analysis. Cancer. 1988;61(1):203-8.

13. Wünsch-Filho V. The epidemiology of oral and pharynx cancer in Brazil. Oral Oncol. 2002;38(8):737-46.

14. Brugère J, Guenel P, Leclerc A, Rodriguez J. Differential effects of tobacco and alcohol in cancer of the larynx, pharynx, and mouth. Cancer. 1986;57(2):391-5.

15. De Stefani E, Boffetta P, Oreggia F, Mendilaharsu M, DeneoPellegrini H. Smoking patterns and cancer of the oral cavity and pharynx: a case-control study in Uruguay. Oral Oncol. 1998;34(5):340-6.

16. MacFarlane GJ, Evstifeeva TV, Scully C, Boyle P. The descriptive epidemiology of pharyngeal cancer in Scotland. Eur J Epidemiol. 1993;9(6):587-90.

Como citar este artigo:

Berto JC, Rapoport A, Lehn CN, Cestari Filho GA, Javaroni AC. Relação entre o estadiamento, o tratamento e a sobrevida no câncer da faringe. Rev Col Bras Cir. [periódico na Internet] 2006 JulAgo;33(4). Disponível em URL: http://www.scielo.br/rcbc

Endereço para correspondência:

Rua Iramaia, 136

Jardim Europa

01450-020 - São Paulo- SP

e-mail: arapoport@terra.com.br 